\title{
Half-spin particles modeled as electromagnetic resonance based micro-black-holes
}

\author{
Thomas A. Kriz \\ Alpha Omega Advanced Studies, 915 Forest Trail, Cedar Park, USA
}

Email address:

t_kriz@yahoo.com

\section{To cite this article:}

Thomas A. Kriz. Half-Spin Particles Modeled as Electromagnetic Resonance Based Micro-Black-Holes. American Journal of Modern Physics. Vol. 4, No. 1, 2015, pp. 10-14. doi: 10.11648/j.ajmp.20150401.12

\begin{abstract}
The energy conversion process in a cosmic expansion scenario, initially in a radiation dominated phase that transits to a half-spin particle matter dominated phase, is characterized as due to an electromagnetic resonance based process with an altered light speed that can be linked to particle based gravitational time dilation. Such a matter formation process is shown to have a space-time signature that is consistent with a General Relativity Schwarzschield micro-black-hole solution. Using Special Relativity as a constraint condition, half-spin particles, when viewed as micro-black-holes, can have the same physically relevant gravitational analytical solutions as General Relativity.
\end{abstract}

Keywords: Energy Conversion, Electromagnetic Resonance, GR Schwarzschield Solution

\section{Introduction}

As shown in a previous paper by our group ${ }^{1}$, the energy conversion process in a cosmic expansion scenario based on a Robertson-Walker model ${ }^{2}$ with an observer constrained by the Stefan-Boltzmann Law and lens-effect distortion due to a spiraling photon trajectory ${ }^{3,4}$ can be characterized as

$$
\left.\mathrm{t}=\mathrm{t}_{\mathrm{d}} \exp \left[\left(\rho_{\mathrm{m}} / \rho_{\mathrm{r}}\right)^{1 / 3}-\rho_{\mathrm{m}} / \rho_{\mathrm{r}}\right)^{1 / 4}\right], \rho_{\mathrm{m}} \geq \rho_{\mathrm{r}}, \mathrm{t} \geq \mathrm{t}_{\mathrm{d}},(1)
$$

where $t$ is current time, $t_{d}$ is decoupling time (eg; the boundary between radiation and matter dominated phases when $\left.\rho_{\mathrm{m}}=\rho_{\mathrm{r}}\right), \rho_{\mathrm{m}}$ is matter density and $\rho_{\mathrm{r}}$ is radiation density at time $\mathrm{t}$, assuming total energy is conserved $\left(\mathrm{eg} ;\left(\rho_{\mathrm{m}}+\rho_{\mathrm{r}}\right)=\mathrm{k}\right.$, a constant). In such a model, where $t_{d}$ is approximately two hundred thousand years, primitive matter (mostly hydrogen and helium) forms rather abruptly at and near decoupling time as compared to that at a later time. The unanswered question, however, is "What is the process by which radiation based photons convert to half spin matter particles, assuming conservation of energy?". Some authors propose that matter has always existed, such as in a static cosmos ${ }^{5}$, or that it exists already during the radiation dominated phase as a plasma ${ }^{6}$. The results in the paper ${ }^{1}$ cited above and a later one by our group ${ }^{7}$, suggest, however, that a half-spin particle can be modeled as one with a special electromagnetic (EM) resonance mode where light speed is internally altered by a gravitational time dilation process caused by the mass of the particle itself. Such a view then allows one to establish a strong link with the general relativity based gravitational attributes of the particle.

\section{A Theoretical Particle Focused EM Resonance Template}

Another paper ${ }^{7}$ also gives evidence that there is a close relationship between quantum waves and EM waves, and furthermore suggests a close relationship between photons and matter. Such a view is also supported by a template ${ }^{1,7}$ that maps theoretical EM resonance properties to matter orbital and spin attributes. This template is developed as follows. Given a harmonic EM waveform propagating in a loss-less medium with frequency $\omega$ and electric field intensity $\mathrm{E}=\mathrm{E}_{0} \mathrm{e}^{\mathrm{i} \omega \mathrm{t}}$ and magnetic field intensity $\mathrm{H}=\mathrm{H}_{0} \mathrm{e}^{\mathrm{i} \omega t}$ where $\mathrm{E}_{0}$ and $\mathrm{H}_{0}$ are field amplitude constants, and $\mathrm{i}=(-1)^{1 / 2}$, the appropriate governing form of Maxwell's Equations is defined by the EM wave equation pair

$$
\begin{gathered}
\nabla^{2} \mathrm{E}+\omega^{2} \epsilon_{0} \mu_{0} \mathrm{E}=0 \text {, and } \\
\nabla^{2} \mathrm{H}+\omega^{2} \epsilon_{0} \mu_{0} \mathrm{H}=0
\end{gathered}
$$

where $\epsilon_{0}$ is the dielectric constant of free space, and $\mu_{0}$ is the 
permeability of free space. Within this framework, the resonance form must also support the appropriate number of EM harmonic nodes required by the orbit or spin to move synchronously with a traveling wave resonance at velocity

$$
\mathrm{v}=\mathrm{c} / \beta,
$$

where $\beta$ is a factor which defines the number of EM full waves (eg; the number of spins), and $c$ is the speed of light. This traveling wave form, when Doppler corrected per Special Relativity, must also present a valid resonance condition, both when looking forward and backward along the traveling wave to yield respectively the observed forward and backward frequencies

$$
\begin{gathered}
\omega_{\mathrm{f}}=\omega[(\mathrm{c}+\mathrm{v}) /(\mathrm{c}-\mathrm{v})]^{1 / 2}, \text { and } \\
\omega_{\mathrm{b}}=\omega[(\mathrm{c}-\mathrm{v}) /(\mathrm{c}+\mathrm{v})]^{1 / 2} .
\end{gathered}
$$

Now, let

$$
\begin{aligned}
& \zeta \omega_{f}=(\beta+1) \omega, \text { (forward resonance), and } \\
& \zeta \omega_{b}=(\beta-1) \omega, \text { (backward resonance). }
\end{aligned}
$$

Reducing the terms in Eqs. $(7,8)$ to an expression which eliminates $\omega$, one then obtains

$$
\zeta^{2}=\beta^{2}\left[1-(\mathrm{v} / \mathrm{c})^{2}\right]=\beta^{2}-1
$$

which defines the relativistic relationship between $\zeta$ and $\beta$ where the constraints on $\beta, \beta \pm 1$, and $v$ are determined by the specific form (eg; orbital, spin, free, etc.) of the resonance condition. It can be seen then that Eq. (9) covers a variety of resonance forms, and may be interpreted to yield a specific theoretical resonance properties list for the following special cases:

- $\beta \rightarrow \infty$, a motionless free electron particle with orbital velocity $v \rightarrow 0$ and $\zeta^{2} \rightarrow \infty$,

- $\beta=N,(N \geq 2 \& N \pm 1$ integers $)$, an orbiting electron particle with $v=c / N<c$, and $\zeta^{2}=N^{2}-1$,

- $\beta=1$, a spin-1 photon particle with velocity $v=c$ and $\zeta^{2}=0$,

- $\beta=1 / 2$, a spin- $1 / 2$ particle with $v=v_{g}=c / 2, v_{p}=c^{2} / v_{g}=2 c$, and $\zeta^{2}=-3 / 4$, and

- $\beta=0$, a meson spin-0 particle with $v=v_{p}=c^{2} / v_{g} \rightarrow \infty$ as $v_{g} \rightarrow 0$, and $\zeta^{2}=-1$,

where $\beta$ is a spin attribute, and $v_{p}$ and $v_{g}$ are the phase wave and group wave velocity respectively of a wave packet focused set of harmonic components. EM based microwave cavity analogs can then be defined to characterize orbiting particles as an $(\beta=N)$ orbital resonance. A tightly closed microwave toroidal loop cavity analog can be invoked to define and characterize photons as a $(\beta=1)$ spin-1 EM resonance; common matter sub-particles as a $(\beta=1 / 2)$ spin- $1 / 2$ EM resonance; and meson particles as a $(\beta=0)$ spin-0 resonance.

\section{Neutron and Decoupling Time Radiation Wavelength}

Photons are known to have an "equivalent mass" property equal to $h v / c^{2}$ where $h$ is Planck's constant, $v$ is the frequency of the photon wave, and $\mathrm{c}$ is the speed of light in a vacuum. ${ }^{8}$ Specifically, a neutron particle (or approximately a Hydrogen atom) with $939.57 \mathrm{MeV} / \mathrm{c}^{2}$, has therefore the equivalent energy of a photon with frequency

$$
v=\mathrm{m}_{\mathrm{n}} \mathrm{c}^{2} / h=2.27 \times 10^{23} \mathrm{~Hz}
$$

or wavelength

$$
\lambda / 2 \pi \approx 1.1 \times 10^{-15} \mathrm{~m}
$$

where $h=4.136 \times 10^{-15} \mathrm{eVs}$. This also happens to be approximately the same as the cosmic background radiation level wavelength at decoupling time $t_{d}$ when

$$
\lambda / 2 \pi \approx 4 \pi\left(\mathrm{t}_{\mathrm{d}} / \mathrm{T}_{\mathrm{p}}\right)^{1 / 3} \mathrm{~L}_{\mathrm{p}} \approx 1.1 \times 10^{-15} \mathrm{~m}
$$

where Planck Time $\mathrm{T}_{\mathrm{p}}=5.39 \times 10^{-44} \mathrm{~s}$ and Planck Length $\mathrm{L}_{\mathrm{p}}=1.616 \times 10^{-35} \mathrm{~m}$ define the initial state, and $t_{d}$ $=2 \times 10^{5} \mathrm{yrs}=6.31 \times 10^{12} \mathrm{~s}$ is the decoupling time. Note here that the leading term of $4 \pi$ in Eq.(12) assumes that cosmic expansion occurs with a volumetric form. Thus, both the concepts of conservation of energy, and conversion of EM waves to matter form are well supported by a close data match in Eqs. (11-12) for neutron equivalent wavelength and the radiation wavelength at decoupling time. A likely radiation to matter conversion scenario then is one where neutrons develop first, followed by protons and electrons that form as a result of Beta decay in an isolated neutron population. ${ }^{1}$ More complex atomic structures then form at a latter stage in aging stellar bodies.

\section{Particle Based Gravitational Time Dilation}

The specific focus of this paper is on the $(\beta=1 / 2)$ spin- $1 / 2$ signature resonance form (eg; $\zeta^{2}=-3 / 4$ ) listed above. The basic question at issue is: How gravitational properties of a matter particle can sufficiently alter light speed so as to be compatible with a $\mathrm{v}=\mathrm{v}_{\mathrm{g}}=\mathrm{c} / 2, \mathrm{v}_{\mathrm{p}}=\mathrm{c}^{2} / \mathrm{v}_{\mathrm{g}}=2 \mathrm{c}$ EM resonance signature? A basic knowledge of modern physics suggests that such an EM signature may be caused gravitational time dilation at the particle itself. Such phenomenon can also be viewed as a form of General Relativity impact that describes the way that particle gravity itself alters local space and time.

The usual context for gravitational time dilation is defined by the gravitational impact of a large body on time dilation at different radial distances from the body. ${ }^{9}$ This paper, however, focuses instead on how a particle based gravitational time dilation process can cause an EM wave to decrease in frequency (and therefore also clock frequency) at the particle level. Such a time dilation impact therefore can constitute an EM resonance trap process for half-spin 
particles as cited above. In such a process, a clock tick (or EM wave frequency) is shifted downward an amount $\Delta v^{9}$

$$
\Delta v / \nu=\Delta \tau / \tau=\mathrm{GM}\left[1 / \mathrm{r}_{1}-1 / \mathrm{r}_{2}\right]
$$

where $v$ is a clock or EM wave frequency, $\Delta \tau / \tau$ is the dilated time, $\mathrm{G}$ is the gravitational constant, $\mathrm{M}$ is the mass of a spherical particle, and $\mathrm{r}$ is the radial position of the wave boundary relative to its origin with $r_{2}>r_{1}$. It can be seen therefore that, when $\Delta v / v=1 / 2$, which corresponds to the EM resonance signature listed above, the spin $1 / 2$ attribute in cgi units is

$$
\Delta v / v=\mathrm{GM} / \mathrm{c}^{2} \mathrm{r}=1 / 2
$$

with a gravitational limit solution

$$
\mathrm{r}=2 \mathrm{GM} / \mathrm{c}^{2} \text {. }
$$

The result in Eq. (15) also corresponds to the well known Schwarzschield solution to the in vacuo Einstein GR field equations that have only non-zero diagonal terms ${ }^{10}$

$$
\mathrm{R}_{\mu v}-1 / 2 \mathrm{~g}_{\mu v} \mathrm{R}=0
$$

where $\mathbf{g}_{\boldsymbol{\mu} \dot{\varepsilon}}$ is the metric tensor, $\mathbf{R}_{\boldsymbol{\mu} v}$ is the Ricci curvature tensor, and $\mathbf{R}$ is the Riemannian scalar curvature tensor. Thus, it can be seen that the gravitational space-time signature with $v=v_{g}=c / 2$ given in Eq.(15) is the same as the half-spin EM resonance based signature given above.

\section{An Alternate Approach to Obtaining the Schwarzschield Solution}

The Schwarzschield solution result traditionally is obtained by solving for Ricci tensor attributes associated with a Riemannian Manifold that defines space curvature, after guessing and checking possible candidate solutions for correctness. ${ }^{11}$ An alternate solution method that greatly reduces complexity, however, is described in another the paper by our group ${ }^{12}$, which regards a space time framework interpretation of Newtonian gravity (eg; A body in free fall over a large mass $\mathrm{M}$ accelerates at $-\mathrm{GM} / \mathrm{r}^{2}$ simply because it's local space-time framework is accelerating toward the large mass at that rate). As shown in that paper, such an analysis approach can also be used to obtain the physically relevant solutions that are available using the traditional GR method mentioned above. In addition to the Schwarzschield solution surrounding a static spherical mass, solutions can also be obtained for gravitational time dilation, gravitational deflection of light, time retardation of light, perihelion precession attributes of an orbiting planet, and a Kerr style field solution for a rotating sphere with mass $M$ and angular momentum $\mathrm{J}$ where radial distance $\mathrm{r}>>\mathrm{J} / \mathrm{M} .{ }^{12}$ It will now be shown here that the same Schwarzschield solution result can also be obtained by invoking Special Relativity as a constraint condition in connection with an analysis of the gravitational tensor attributes.

\section{Schwarzschield Solution using Special Relativity as a Constraint Condition}

As indicated in the text by Ohanian and Ruffini ${ }^{12}$, there is no relativity principle that is more general than Special Relativity. The term "General Relativity" used to describe the impact of gravity on the space-time continuum is actually subservient to Special Relativity, and does not define a more general relativity principle. This suggests the possibility of a GR solution approach that uses Special Relativity as a constraint condition. This possibility is demonstrated in the following. To begin, define the Newtonian inverse-square law of gravity as follows ${ }^{13}$

$$
a=\mathrm{d} v / \mathrm{dt}=-\mathrm{Gmr}{ }^{2}=\mathrm{dr} / \mathrm{dt} \cdot \mathrm{d} v / \mathrm{dr}=v \mathrm{~d} v / \mathrm{dr}
$$

where $a$ is the acceleration of the body in free fall in the modified Newtonian gravity mentioned above, $v$ is velocity of the space-time framework, $r$ is radial distance, and $t$ is time. Now let

$$
\int_{0+\infty}^{\substack{\mathrm{r} \\ v \mathrm{~s}}} \mathrm{~d} v=\int-\mathrm{Gmr}^{2}=\mathrm{c}^{2} / 2=\mathrm{Gm} / \mathrm{r}_{\mathrm{s}}
$$

where it can be seen that $r_{s}=2 \mathrm{Gm} / \mathrm{c}^{2}$ is a micro-black-hole solution. Eqs. $(17,18)$ also imply a general relation

$$
\mathrm{rv}^{2}=\mathrm{r}_{\mathrm{s}} / \mathrm{c}^{2}=2 \mathrm{Gm}
$$

which when combined with the Newtonian gravitational potential $\Phi=-\mathrm{Gm} / \mathrm{c}^{2}$ (in cgs units) yields

$$
\mathrm{v}^{2} / \mathrm{c}^{2}=\mathrm{r}_{\mathrm{s}} / \mathrm{r}=2 \mathrm{Gm} / \mathrm{c}^{2} \mathrm{r}=-2 \Phi .
$$

An equally valid alternative form of Eq.(19) can then also be written as 13

$$
1-\mathrm{v}^{2} / \mathrm{c}^{2}=1-\mathrm{rs} / \mathrm{r}=1-2 \mathrm{Gm} / \mathrm{c}^{2} \mathrm{r}=1+2 \Phi .
$$

Thus, the above micro-black-hole solution is consistent with a Special Relativity constraint $\left(1-\mathrm{v}^{2} / \mathrm{c}^{2}\right)$.

Exploring further, it may be noted that a metric tensor for space-time in Eq.(21) can be defined by a linear form metric tensor

$$
\mathbf{g}_{\mu \nu}=\boldsymbol{\eta}_{\mu \nu}+\mathbf{X}_{\mu \nu}
$$

where

$$
\boldsymbol{\eta}_{\mu \nu}=[1,0,0,0 ; 0,-1,0,0 ; 0,0,-1,0 ; 0,0,0,-1]
$$

is a flat space-time metric tensor component (using the matrix form notation [row1; row2;row3;row4], and

$$
X_{\mu \nu}=\left[\mathrm{f}_{1}(\Phi), 0,0,0 ; 0, \mathrm{f}_{2}(\Phi), 0,0 ; 0,0, \mathrm{f}_{2}(\Phi), 0 ; 0,0,0, \mathrm{f}_{2}(\Phi)\right]
$$

is a curved space-time metric tensor component (using the same matrix form notation) to characterize the impact of Newtonian gravitational potential, $\Phi$ on $\mathbf{g}_{\mu \nu}$. The terms, $f_{l}(\Phi)$ and $f_{2}(\Phi)$, in Eq. (24) are unknown functions of $\Phi$. The specific form of the space-time interval line element $d s$ for the metric $g_{\mu \nu}$ can then be obtained using the general 
definition of a space-time interval line element $d s$ as: ${ }^{14}$

$$
\mathrm{ds}^{2}=\mathrm{g}_{\mu \nu} \mathrm{dx} \mathrm{x}^{\mu} \mathrm{dx} \mathrm{x}^{\nu}=\mathrm{d} \tau^{2}-\mathrm{dl^{2 }}
$$

where the line element $d \tau$ defines proper time and line element $d l$ defines proper local distance. In this case, the tensor components in Eq. (22) yield the space-time interval line element $d s$ expression

$$
\mathrm{ds}^{2}=\left[1+\mathrm{f}_{1}(\Phi)\right] \mathrm{dt}^{2}-\left[1-\mathrm{f}_{2}(\Phi)\right]\left(\mathrm{dx}^{2}+\mathrm{dy}^{2}+\mathrm{dz}^{2}\right)
$$

where $d \tau^{2}=\left[1+f_{l}(\Phi)\right] d t^{2}$ term corresponds to a time-like geodesic component (eg; $\left.d l^{2} \approx 0\right)$ and $d l^{2}=[1-$ $\left.f_{2}(\Phi)\right]\left(d x^{2}+d y^{2}+d z^{2}\right)$ corresponds to a space-like geodesic component (eg; $\mathrm{d} \tau^{2} \approx 0$ ). Invoking a Special Relativity time dilation based constraint via the equation sequence in Eq.(21) the time-like geodesic component, $d l^{2}=\left[1+f_{l}(\Phi)\right] d t^{2}$ then becomes which indicates that $f_{l}(\Phi)=2 \Phi=-2 G \mathrm{~m} / \mathrm{c}^{2} r$ for a spherical mass. Then, if one assumes that $f_{l}(\Phi)=f_{2}(\Phi)$, Eq. (27) corresponds to a linear form GR analog of Maxwell's Equations, and becomes ${ }^{15}$

$$
\mathrm{ds}^{2}=\left(1-2 \mathrm{Gm} / \mathrm{c}^{2} \mathrm{r}\right) \mathrm{dt}^{2}-\left(1+2 \mathrm{Gm} / \mathrm{c}^{2} \mathrm{r}\right)\left(\mathrm{dx}^{2}+\mathrm{dy}^{2}+\mathrm{dz}^{2}\right) \text {. }
$$

Eq. (28) then implies a linear form GR space-time metric (which can be used with less analytical complexity to obtain solution of problems not requiring full use of a Schwarzschild geometry ${ }^{15}$

$$
\mathbf{g}_{\mu \nu}=\boldsymbol{\eta}_{\mu \nu}+\mathbf{k} h_{\mu \nu}
$$

where

$\boldsymbol{\kappa} h_{\mu \nu}=[-2 \mathrm{Gm} / \mathrm{r}, 0,0,0 ; 0,-2 \mathrm{Gm} / \mathrm{r}, 0,0 ; .0,0,-2 \mathrm{Gm} / \mathrm{r}, 0 ; 0,0,0,-2 \mathrm{Gm} / \mathrm{r}]$.

Note also that the speed of light in Eq. (30) has been analytically forced to be unity. Next, if one considers the form of the space-like geodesic (where $d t^{2} \approx 0$ ) implied by Eq. (22), it can be seen that when $d t^{2} \approx 0$

$$
\mathrm{ds}^{2}=\left(1+2 \mathrm{Gm} / \mathrm{c}^{2} \mathrm{r}\right) \mathrm{d} l^{2} \approx\left(1+\mathrm{v}^{2} / \mathrm{c}^{2}\right) \mathrm{d} l^{2}
$$

The connection between Eq. (31) and Special Relativity may not be immediately obvious. However, if one invokes an analytical expansion of the Special Relativity expression

$$
\left(1-v^{2} / c^{2}\right)^{-1}=1+v^{2} / c^{2}+\left(v^{2} / c^{2}\right)^{2}+\left(v^{2} / c^{2}\right)^{3}+\ldots,
$$

it can be seen that $\left(1+v^{2} / c^{2}\right)$ corresponds to the first two terms of an expansion for $\left(1-v^{2} / c^{2}\right)^{-1}$. Thus, Eq. (32) constitutes a linear approximation of the more accurate space-like geodesic expression

$$
\mathrm{ds}^{2} \approx\left(1-\mathrm{v}^{2} / \mathrm{c}^{2}\right)^{-1} \mathrm{~d} l^{2}=(1+2 \Phi)^{-1} \mathrm{~d} l^{2}=\left[1-\mathrm{f}_{2}(\Phi)\right] \mathrm{d} l^{2}
$$

Special Relativity consistency can then be completely assured if the metric tensor in Eq. (22) is instead revised to be

$$
\mathbf{g}_{\mu v}=\left[2 \Phi, 0,0,0 ; 0,(1+2 \Phi)^{-1}, 0,0 ; 0,0,(1+2 \Phi)^{-1}, 0 ; 0,0,0,(1+2 \Phi)^{-1}\right](34)
$$

Evaluating Eq.(22) in terms of Eq. (34) with $2 \Phi=-2 G m / c^{2} r$ for a spherical mass, one then obtains

$$
\mathrm{ds}^{2}=\left(1-2 \mathrm{Gm} / \mathrm{c}^{2} \mathrm{r}\right) \mathrm{dt}^{2}-\left(1-2 \mathrm{Gm} / \mathrm{c}^{2} \mathrm{r}\right)^{-1}\left(\mathrm{dx}^{2}+\mathrm{dy}^{2}+\mathrm{dz}^{2}\right),
$$

or equivalently in $(r, \Theta, \Phi)$ spherical coordinates

$$
\mathrm{ds}^{2}=\left(1-2 \mathrm{Gm} / \mathrm{c}^{2} \mathrm{r}\right) \mathrm{dt}^{2}-\left(1-2 \mathrm{Gm} / \mathrm{c}^{2} \mathrm{r}\right)^{-1} \mathrm{dr}^{2}-\mathrm{r}^{2}\left(\mathrm{~d} \theta^{2}+\sin ^{2} \theta \mathrm{d} \Phi^{2}\right),
$$

which is the same as the Schwarzschild solution from GR for a static spherical mass. It should be noted that this result was obtained explicitly using Special Relativity as a constraint instead of the usual GR approach. The Schwarzschild solution in Eq. (36) then provides a basis for one to obtain other physically relevant GR style solutions as listed above. 13

\section{Discussion and Conclusions}

The forgoing analytical scenario gives a credible basis for converting photon energy to matter form via an EM resonance based mode that depends upon particle based gravitational time dilation. As a initial point of focus, it is shown that an energy based comparison of neutron attributes with cosmic decoupling time wavelength is in close agreement, thereby giving evidence to support both conservation of energy, and transformation of radiation to matter form as basic cosmology concepts. A theoretical template for various particle based EM resonance forms was then developed to show that there should also be an EM resonance signature that corresponds to that of a half spin matter particle. It was also shown analytically then that such a signature can be physically achieved at the particle itself if one invokes particle based time dilation as the cause of an altered space-time attribute that corresponds to the EM resonance signature form.

Somewhat surprisingly, this result also corresponds to a particle level Schwarzschild solution from GR with a very small spherical mass (eg; a micro-black-hole). It should be noted that, since such a micro-black-hole is finite but small in size, it can be related to the point mass and point concepts that are commonly found both in both classical physics and quantum theory. However, in quantum electrodynamics (QED) theory, when developing a QM perturbation expansion result, point size can lead to unwanted analytical infinities in the higher order terms because of an implied zero distance between the perturbation series terms. ${ }^{16,17}$ The analytical interaction between a charged particle and a photon was resolved, in theory, by Feynman and others using analytical regularization methods to subtract out the unwanted infinities, ${ }^{18-20}$ but later repudiated by Feynman as a "hocus-pocus" and "shell game" process. ${ }^{21}$ A more recent paper by our group ${ }^{22}$, however, avoids the dilemma by invoking the use of a microwave analog method in the QED analysis approach. The foregoing also shows how the Schwarzschild solution can be obtained by invoking Special Relativity as a general relativistic constraint condition..

It is also important to note that particle based micro-blackholes are different than stellar black holes supported by a large mass collective. Whereas stellar black-holes have no 
observable internal structure, both cosmological and quantum theory give evidence that an internal structure in particle micro-black-holes is necessary to support wavelike and atomic properties of particle based matter. Moreover, although particle micro-black-holes can also collectively coalesce as atomic nuclei, they do not otherwise exhibit the gravitational stellar black-hole appetite to swallow up other nearby matter. ${ }^{16,17}$ Neutron micro-black-hole collections as an isolated particle set are also known to be unstable. ${ }^{16,17}$

\section{Acknowledgements}

The author wishes to express gratitude for the encouragement and critical review received during the early stages of the work discussed here from E.J. Bacinich, Director of the Alpha Omega Research Foundation, and several other members of our group, now deceased: Prof. Behram Kursunoglu and Dr. Walter Rosenthal. Prof. Kursunoglu was the Director of the Theoretical Institute at the University of Miami (FL), and pursued graduate studies at Cambridge University under P.A.M. Dirac. Dr. Rosenthal was one of the last students to pursue graduate studies in physics at the University of Berlin under Max Planck.

\section{References}

[1] T.A. Kriz, and E.J. Bacinich, Phys. Essays 21, 273 (2008).

[2] H.C. Ohanian, and R. Ruffini, Gravitation and Spacetime (second edition), (Norton \& Co, New York, NY and London 1994), pp.543-52.

[3] E.J. Bacinich and T.A. Kriz, Hubble Expansion Caused Distortion in Cosmological Observations, GR13 Abstracts, Int. Conf. on Gravity and GR., Cordoba, Argentina (1992).

[4] E.J. Bacinich, and T.A. Kriz, Phys. Essays 8, 508 (1995).

[5] F. Hoyle, G. Burbidge, J.V. Narlikar , "A quasi-steady state cosmological model with creation of matter", The Astrophysical Journal 410: 437-457 (1993).

[6] H.C. Ohanian, and R. Ruffini, Gravitation and Spacetime (second edition), (Norton \& Co, New York, NY and London 1994), Ch 10.
[7] T.A. Kriz, and E.J. Bacinich, Phys. Essays 22, 581 (2009).

[8] A. Beiser, Concepts of Modern Physics (fifth edition), (McGraw-Hill, New York 1995), p. 387.

[9] H.C. Ohanian, and R. Ruffini, Gravitation and Spacetime (second edition), (Norton \& Co, New York, NY and London 1994), p. 167-171.

[10] H.C. Ohanian, and R. Ruffini, Gravitation and Spacetime (second edition), (Norton \& Co, New York, NY and London 1994), Ch. 7.4.

[11] R.M. Wald, General Relativity, (University of Chicago Press, Chicago IL 1984), Ch 6.

[12] H.C. Ohanian, and R. Ruffini, Gravitation and Spacetime (second edition), (Norton \& Co, New York, NY and London 1994), p. 377.

[13] T.A. Kriz, Phys. Essays 24, p.124 (2011).

[14] H.C. Ohanian, and R. Ruffini, Gravitation and Spacetime (second edition), (Norton \& Co, New York, NY and London 1994). p.322.

[15] H.C. Ohanian, and R. Ruffini, Gravitation and Spacetime (second edition), (Norton \& Co, New York, NY and London 1994). Ch. 3.2.

[16] P.J.E. Peebles, Quantum Mechanics, (Princeton University Press, 1992), Ch. 5.

[17] R.P. Feynman, QED - The Strange Theory of Light and Matter, (Princeton University Press, 1985), p.129.

[18] R.P. Feynman, Phys. Rev. 76 (6): pp.749-759, (1949).

[19] R.P. Feynman, Phys. Rev. 76 (6): pp.769-789, (1949).

[20] R.P. Feynman, Quantum Electro-dynamics, (Addison-Wesley Publishing Co., New York 1961).

[21] R.P. Feynman, QED - The Strange Theory of Light and Matter, (Princeton University Press, 1985), p.128.

[22] T.A. Kriz, Phys. Essays 25, p.221 (2012). 